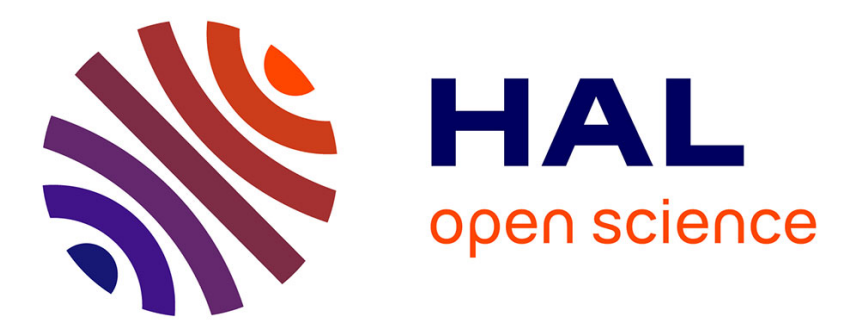

\title{
Vers de nouveaux modes de gouvernement des systèmes éducatifs? Les leçons des comparaisons internationales
}

Denis Meuret, Marie Duru-Bellat

\section{To cite this version:}

Denis Meuret, Marie Duru-Bellat. Vers de nouveaux modes de gouvernement des systèmes éducatifs ? Les leçons des comparaisons internationales. Reconfigurer l'action publique: big bang ou réforme? Onzième colloque international, Jun 2002, Nice, France. pp.61-79. halshs-00004864

\section{HAL Id: halshs-00004864 \\ https://shs.hal.science/halshs-00004864}

Submitted on 8 Jun 2021

HAL is a multi-disciplinary open access archive for the deposit and dissemination of scientific research documents, whether they are published or not. The documents may come from teaching and research institutions in France or abroad, or from public or private research centers.
L'archive ouverte pluridisciplinaire HAL, est destinée au dépôt et à la diffusion de documents scientifiques de niveau recherche, publiés ou non, émanant des établissements d'enseignement et de recherche français ou étrangers, des laboratoires publics ou privés.

\section{(ㅇ)(1) $\$$}

Distributed under a Creative Commons Attribution - NonCommercial - NoDerivatives 44.0 


\title{
VERS DE NOUVEAUX MODES DE GOUVERNEMENT DES SYSTEMES EDUCATIFS ? LES LEÇONS DES COMPARAISONS INTERNATIONALES
}

\author{
Denis MEURET* \\ Marie DURU-BELLAT*
}

Résumé

\begin{abstract}
Dans la plupart des pays développés, l'autonomie des établissements scolaires s'est accrue, accompagnée en général par une surveillance de leurs performances et par des mécanismes d'incitation. Par ailleurs, les parents peuvent davantage choisir l'école de leurs enfants. Cela s'est passé en France aussi, mais de façon plus retenue, et sans que le mode de régulation du système éducatif n'évolue en conséquence. Cet article présente les politiques implantées à l'étranger en la matière, en particulier au Royaume-Uni et dans certains Etats ou Districts des Etats-Unis, et le bilan que l'on peut faire des politiques de choix de l'école comme des politiques d'autonomie/régulation par les résultats. II présente ensuite l'évolution du système éducatif français en ce domaine et le choix qui a été fait d'une régulation par les processus plutôt que par les résultats. Tout en relevant que certains aspects du système français sont en fait repris par le modèle vers lequel convergent la plupart des systèmes éducatifs, et que les performances du système français souffrent la comparaison avec celle des systèmes anglo-saxons, il souligne les effets délétères de ses ambiguïtés sur les personnels et sur les relations qu'ils entretiennent avec les élèves ou leurs familles. L' "exception française" en matière de régulation est interprétée comme l'effet de la prévalence d'un modèle dans lequel l'éducation institue la république et la citoyenneté plutôt qu'elle n'est au senvice des individus et de la collectivité.
\end{abstract}

\footnotetext{
* Université de Bourgogne-IREDU-CNRS.

Revue POLITIQUES ET MANAGEMENT PUBLIC, Volume 20, n², juin 2002.

(C) Institut de Management Public - 2002.
} 
Au cours des vingt dernières années, deux évolutions ont affectè la plupart des systèmes éducatifs, d'une part le développement de l'autonomie des établissements scolaires, accompagné d'une surveillance accrue de leur efficacité, d'autre part, l'expansion du choix de l'école par les parents' 1 . Auparavant, la situation la plus fréquente ètait qu'une administration édictait des règles dont la mise en œuvre faisait l'objet d'un contrôle de conformité. Ces évolutions misent toutes deux sur la responsabilité des individus et accroissent leur marge de manceuvre : celle du personnel des établissements dans le cas de l'autonomie, celle des usagers dans le cas du choix. Elles représentent une inflexion majeure de la régulation des systèmes éducatifs, que nous entendons ici comme la manière dont une instance publique s'y prend pour orienter les comportements des acteurs sur lesquels elle a autoritè.

Cependant, ces évolutions ont pris des formes différentes selon les pays. Le Royaume-Uni, la Nouvelle Zélande, l'Australie, certains Etats américains, se sont engagés résolument dans cette voie, notre pays, avec à la fois prudence et réticence. La solution française peut être appréciée de différentes façons, qui renvoient aux différentes théories qui ont été élaborées pour rendre compte de la convergence des systèmes éducatifs (Schriewer, 1997). Cette convergence peut être interprétée comme l'effet de la domination d'un groupe de pays sur les autres - auquel cas la singularité française serait l'effet d'une résistance légitime à cette domination -, comme l'effet d'une rationalisation progressive de l'ensemble des différents systèmes - auquel cas la singularité française peut être lue soit comme une résistance fondée sur une tradition caduque, soit comme une hybridation réussie entre la tradition locale et la rationalisation globale. Nous voudrions ici tenter d'apprécier la situation française, en présentant le contexte politique de l'évolution des modes de régulation de l'éducation, puis cette évolution elle même et le bilan que l'on peut en faire, d'abord dans les pays où elle a été le plus radicale, puis en France.

\section{Le contexte politique des changements dans la régulation de l'éducation}

Autonomie et choix des écoles se sont développés dans un contexte d'insatisfaction vis à vis des systèmes publics d'éducation, plus marquée d'ailleurs dans les pays anglo-saxons qu'en France. Dans tous les pays développés, la scolarisation a connu après guerre une très forte expansion, accompagnée de réformes de structure dominées par la "bonne forme" de l'"école moyenne", établissement de premier cycle du secondaire fréquenté par toute une génération. Progressivement, des voix se sont élevées contre cette standardisation, jugèe inefficace et incapable de démocratiser véritablement l'institution. Cette défiance s'inscrit aussi dans la remise en cause de l'État providence. Le caractère bureaucratique des systèmes éducatifs publics est, pour certains (Chubb and Moe, 1990), la source même de leur inefficacité.

Une première réaction des pouvoirs publics fut de reconsidérer les prérogatives des différents échelons politiques. Une décentralisation s'est produite dans les pays centralisés (Suède, par exemple), et une certaine centralisation dans les pays décentralisés (Royaume-Uni, par exemple), de

\footnotetext{
1 Nous abordons ici seulement la régulation de l'enseignement primaire et secondaire.
} 
sorte qu'on assiste à une relative convergence vers un modèle dans lequel l'État a en charge les objectifs de l'éducation (les programmes d'études) et la vérification qu'ils sont atteints (examens, évaluations), mais où la gestion des moyens est fortement décentralisée.

Une seconde réaction, qui est l'objet propre de ce texte, a consisté à redéfinir les responsabilités respectives de l'administration, des parents et des professionnels dans le fonctionnement des établissements scolaires. De cette évolution, ses promoteurs attendent :

- Une plus grande responsabilité des acteurs

- Une plus grande efficacité. Certains l'attendent de l'action des professionnels et d'autres de celle des usagers. Les premiers considèrent que les enseignants qui sont au contact des èlèves sont les mieux à même d'adapter l'enseignement à leurs besoins et que, étant plus autonomes, ils seront aussi plus motivés. Les seconds estiment que les enseignants ne rechercheront l'efficacité que s'ils y sont poussés par les usagers, dont la pression sera plus forte dans les ètablissements autonomes.

- Davantage de diversité de l'offre d'éducation, soit dans une logique de marché, attentive aux demandes des familles, soit dans une logique communautaire, attentive aux revendications des communautés

- Davantage de démocratie. Dans les pays nordiques, l'autonomie de l'école est perçue comme un moyen d'associer les élèves eux-mêmes aux décisions ; ailleurs, l'idèe de démocratie évoque plutôt l'implication des parents dans la vie de l'école, pour que celle-ci cesse d'être sous la coupe exclusive des professionnels.

Quatre arguments principaux ont été avancés contre cette évolution :

- L'État se défausse ainsi de ses responsabilités dans les difficultés des systèmes éducatifs en les attribuant aux écoles et aux enseignants.

- L'autonomie professionnelle des enseignants est mise en cause, par l'accroissement de l'influence soit des parents (dotés de plus de choix), soit de la bureaucratie (par l'apparition de programmes ou d'évaluations nationaux).

- Contraints par une surveillance plus étroite de leurs résultats, les enseignants se centrent sur les compétences évaluées, au détriment d'autres objectifs importants de l'éducation.

- Surtout, toutes ces réformes avantagent les classes moyennes et les établissements scolaires que leurs enfants fréquentent, accroissant ainsi les inégalités sociales devant l'éducation.

Il est intéressant de considérer plus attentivement l'environnement idéologique de ces deux politiques d'autonomie et de choix au Royaume-Uni et aux Etats Unis, à la fois parce que les réformes y ont été plus radicales qu'ailleurs, et 
parce que les recherches sur les effets des nouvelles méthodes de régulation y sont nombreuses.

Au Royaume-Uni, l'Education Act de 1988 encourage le choix par la libéralisation des règles d'inscription dans les ècoles. Par ailleurs, il instaure aussi le Local Management of Schools, qui confère aux écoles une grande autonomie en matière de gestion du personnel et des ressources. Ces diverses mesures ne vont pas jusqu'à instaurer un "vrai " marché, - le financement de l'éducation demeure public - mais l'objectif est bien de libérer les initiatives des écoles et la responsabilité des parents. Cette politique a été conservée, mais infléchie par le gouvernement travailliste après son retour au pouvoir : les Local Education Authorities (LEA) ont retrouve un rôle important, le choix des élèves par les écoles a été davantage encadré, etc. De fait l'Angleterre propose aujourd'hui une combinaison originale de mécanismes de marché et de régulation institutionnelle forte (Finkelstein et Grubb, 2000), qui s'exprime dans cette règle: "L'autonomie de chaque école sera proportionnelle à son succès ".

Aux États-Unis, le développement de l'autonomie comme celui du choix prend la forme de réformes locales. Ceci dans un contexte très marqué par le rapport "A Nation at Risk" (1983), qui dressait un tableau dramatique des risques que la mauvaise qualité de leurs systèmes éducatifs faisait courir aux Etats-Unis. Aujourd'hui, 49 Etats fixent des standards (un niveau de compétence que les élèves de tel âge doivent maîtriser), tous testent les compétences de leurs élèves, 27 tiennent les établissements pour responsables de leurs résultats (Education Week, 2001). Le "libre choix" est moins répandu. II est défendu par une mouvance idéologique hétérogène : des libéraux qui prônent plus de marché et moins d'Etat, mais aussi des conservateurs, qui défendent l'implication de l'Etat comme garant de valeurs académiques et morales (Carl, 1994) et aussi quelques responsables politiques "de gauche" qui y voient un moyen de donner plus de pouvoir aux familles populaires et d'en finir avec la "sélection par les loyers" (Viteritti,1999).

Les politiques mises en œuvre
Distinguons les politiques qui jouent sur l'offre d'enseignement (autonomie des écoles, réformes du mode de tutelle) de celles qui jouent sur la demande (liberté du choix de l'école), bien qu'elles conjuguent évidemment leurs effets : en Angleterre, l'inspection aurait moins d'effet sur les établissements si ses rapports n'étaient pas accessibles à des parents disposant de la possibilité de choisir.

\section{Politiques d'offre}

Au Royaume-Uni, les établissements bénéficient d'une grande autonomie de gestion, y compris dans le recrutement du personnel. Aux Etats-Unis, la plupart des établissements secondaires ne bénéficient pas d'une autonomie plus grande que leurs homologues français (OCDE,1998). Cependant, certains districts ont développé le "School Based Management"(SBM), moins répandu, et aussi moins radical que son équivalent anglais. 
Dans ces deux pays, l'autonomie accrue des établissements s'est accompagnée d'une modification, et non d'un allègement, de la tutelle administrative. Davantage tournée vers les résultats, elle prend deux formes essentiellement, soit l'inspection des établissements, soit une combinaison de mesure des résultats, d'aide et d'incitations reçues de l'administration de tutelle (accountability).

Au Royaume-Uni, les ètablissements sont inspectés tous les six ans par des agents de l'OFSTED, qui rencontrent les parents, puis examinent l'ensemble de la vie de l'établissement, observent l'enseignement, selon des critères publics, écrivent ensuite deux rapports, l'un public et l'autre destiné à l'école. auquel elle doit répondre en indiquant comment elle entend remédier aux critiques qui lui sont faites. II n'existe pratiquement pas d'Inspection aux Etats Unis.

En Angleterre, tous les élèves sont testés à 7, 11 et 14 ans sur les matières du programme national. Des objectifs ont été fixés quant au niveau que devaient atteindre en 2002 les élèves de 11 ans en anglais et en mathématiques, un plan d'ensemble a été conçu pour les atteindre, et le Ministre s'est engagé à démissionner en cas d'èchec. Ces objectifs sont déclinés par établissement.

Fixer des objectifs est peu fréquent aux Etats-Unis, mais les Etats multiplient les épreuves de fin d'année, et les comparaisons des résultats, entre Etats, entre écoles d'un même Etat. Une loi récente subordonne l'octroi des subventions fédérales aux Etats à l'existence de telles épreuves, annuelles, pour tous les élèves de 9 à 15 ans. Des récompenses peuvent être attribuées aux établissements en fonction des performances moyennes de leurs élèves, ou de leurs élèves appartenant à des minorités, en fonction aussi de l'évolution de ces performances. Dans quelques districts, lorsqu'un établissement est trop peu "performant", il entre dans un programme de "soutien", et, en cas d'échec, l'ensemble de son personnel est remplacé (Reconstitution). En Floride, la sanction est l'autorisation donnée aux élèves de la zone de recrutement des écoles qui ont durablement de mauvais résultats d'aller dans une autre école publique, ou de recevoir un "voucher" pour aller dans le privé (Herrington et Kasten, 2000).

L'équivalent anglais de ces incitations négatives est la procédure dite "Fresh Start", par laquelle l'établissement passe sous la tutelle directe du Ministère, et doit appliquer un plan de redressement radical, si l'OFSTED a jugé par deux fois que la qualité de son enseignement était inférieure au minimum admissible.

\section{Politiques de demande}

Certaines politiques de "choix de l'école" consistent à financer les écoles privees, ainsi rendues aussi accessibles que les écoles publiques. Nous nous intéresserons ici surtout aux politiques qui affectent les règles d'inscription au sein du secteur public. Elles revêtent des formes variables : la notion de zone de recrutement peut être supprimée ou seulement assouplie; on peut donner plus ou moins de latitude aux écoles pour sélectionner leurs élèves; la 
différenciation et la compétition entre les écoles peut être plus ou moins encouragée; une information indépendante sur leur qualité peut être ou non diffusée.

La liberté de choix existe depuis longtemps dans un certain nombre de pays européens. C'est le cas en Belgique, où existe un "quasi marché" avec à la fois des financements publics et le libre choix de l'école (Vandenberghe, 1997). C'est le cas aussi aux Pays-Bas (Karsten, 1994; Teelken, 1999), où le choix de l'école est inscrit dans la constitution : toutes les familles doivent pouvoir trouver une école, financée par les fonds publics, permettant de satisfaire leurs valeurs culturelles et religieuses. Dans ces deux pays, cette liberté est depuis quelque temps davantage utilisée dans une perspective «consumériste», elle est aussi davantage encadrée par des règlements étatiques.

En Angleterre, les parents peuvent s'adresser directement à certaines écoles (ex-Grant Maintained Schools, écoles religieuses), mais, pour les autres, de loin les plus nombreuses, ils émettent un vœu auprès de l'administration locale (LEA). Celle ci doit leur donner satisfaction s'il reste de la place dans l'école demandée. En cas d' "oversubscription", des règles, depuis 1998, encadrent les procédures d'admission: pas de recrutement selon le niveau scolaire des candidats, les critères doivent être publics et clairs, etc.

Les Etats-Unis illustrent la variété des systèmes de choix possibles. Alors que prévaut le plus souvent un système de type carte scolaire pour le recrutement des écoles publiques ${ }^{1}$ quelques districts ont expérimenté des systèmes de choix, qui diffèrent par le degré d'"encadrement" des choix des familles d'une part, le degré de liberté laissé aux écoles d'autre part.

Dans certains cas, il s'agit de systèmes de choix contrôlé, dans lesquels l'administration scolaire est très présente. Certains valorisent explicitement l'équité. Ainsi, à Minneapolis (Glazerman, 1998), un système de choix existe depuis 1970, initialement instauré pour lutter contre la ségrégation raciale. II concerne la totalité des écoles publiques, et, dès l'entrée du primaire, tous les parents doivent faire trois vœux. L'administration affecte les élèves selon leur choix, tout en veillant à ce que les écoles ne s'écartent pas de plus de $15 \%$ de la proportion élèves blancs/non blancs du district. Quand, pour une école, les demandes excèdent les capacités d'accueil, on procède par tirage au sort, une formule d'ailleurs fréquente dans ces systèmes.

Une forme plus radicale, bien connue mais en réalité peu fréquente, est celle des "vouchers». En théorie, il s'agît d'organiser un marché où les consommateurs pourraient choisir librement, seul le financement restant public. L'Etat conserve un triple rôle : dispenser les subsides, agréer les écoles et faire fonctionner le tout en s'assurant que l'information sur les écoles circule. en vérifiant que tous les enfants ont accès à une école agréėe. II peut aussi instaurer une discrimination positive, en donnant à certains enfants des vouchers de plus grande valeur. Ainsi, le dispositif mis en place à Milwaukee depuis 1990 s'adresse uniquement aux enfants de milieu défavorisé, à qui il

$112 \%$ des élèves fréquentent le privé payant. 
permet de fréquenter gratuitement des écoles privées payantes. On pourrait multiplier les exemples illustrant la variété des systèmes de choix mis en place aux Etats-Unis. Une chose est sûre, de nombreux systèmes peuvent être imaginés, entre le choix intégral non régulé et la carte scolaire la plus rigide.

\section{L'évaluation des politiques d'autonomie et de choix ${ }^{1}$}

L'analyse des politiques publiques consiste souvent à les confronter à un idéal plus ou moins inspiré de théories économiques ou sociologiques sur le fonctionnement des organisations. A cet égard, les systèmes de régulation que nous venons d'évoquer peuvent s'autoriser de théories économiques comme le "public choice» ou la théorie "Principal/agent» (Meuret et Duru-Bellat, 2001).

Nous préférons ici risquer une évaluation de ces politiques par leurs résultats En effet, les données disponibles dans le champ de l'éducation permettent, dans une certaine mesure, de s'appuyer sur des mesures empiriques de l'efficacité ou de l'équité de ces politiques.

\section{Les effets de l'autonomie des établissement et de la régulation par les résultats}

On se demandera d'abord si, au sein d'un pays donné, les élèves apprennent mieux dans les établissements autonomes que dans les autres, puis si, dans les pays qui ont implanté fortement ces politiques, l'efficacité de l'enseignement s'est accrue.

De la littérature américaine, il ressort (Murphy et Beck, 1995) que les enseignants participent davantage à la vie des établissements autonomes, mais que cette participation accrue ne se traduit pas par des changements dans l'enseignement. Et la plupart des chercheurs concluent que l'autonomie ne permet pas d'améliorer l'efficacité (Leightwood et Menzies, 1998). On trouve néanmoins quelques évaluations plus positives. Ainsi, Lee et Smith (1995) montrent que les établissements "restructurés" présentent des progressions plus importantes, des progressions plus indépendantes de l'origine sociale des élèves, et un meilleur engagement des élèves dans l'activité d'apprentissage. Toutefois, les établissements restructurés sont des établissements qui ont effectivement utilisé leur autonomie pour mettre en œuvre des pratiques plus efficaces.

Ceci suggère que l'autonomie doit être accompagnée d'incitations à l'efficacité. Le fait que les procédures appliquées aux écoles en difficulté ("Nouveau départ ", "Reconstitution") produisent des effets plutôt positifs, non pas dans tous, mais dans un bon nombre de cas, va dans le même sens.

De plus, une recherche récente (Wössmann, 2000), fondée sur la troisième ètude internationale sur les maths et les sciences (sur les élèves de 13 ans de 40 pays observés en 1995) montre que l'autonomie des établissements est

1 On trouvera un exposé plus détaillé de cette évaluation in Duru-Bellat M. et Meuret D. (2001) 
associée à davantage d'efficacité quand elle porte sur la gestion des moyens (recrutement et salaire des enseignants), mais à moins d'efficacité quand elle porte sur le contenu des programmes. C'est le fait que ces derniers soient fixés au niveau central qui favorise l'efficacité Cette étude plaide, elle aussi, pour un encadrement de l'autonomie des établissements par une attention de la tutelle à leurs résultats.

Les comparaisons longitudinales ont l'inconvénient de mesurer l'effet de tous les changements survenus dans la période, et pas seulement les effets des politiques évaluées. Notons néanmoins qu'au Royaume-Uni, les résultats à l'examen du GCSE se sont améliorés, et nettement plus rapidement depuis qu'ont été implantés les changements étudiés ici. Quant aux tests qui mesurent les compétences aux âges clés, les évolutions récentes sont positives en Anglais et en Maths, moins en Sciences. On peut donc conclure dans le sens d'une amélioration du niveau des élèves en Angleterre depuis ces réformes, cependant ni très forte, ni générale ${ }^{1}$.

Aux Etats Unis, en lecture et en expression écrite, les scores moyens, comme les inégalités entre groupes ethniques, sont restés stables de 1984 à 1996 (Conditions of Education, 1998). En revanche, en Maths et en Science, les scores moyens des élèves blancs ont augmenté selon les âges de 3 à $6 \%$, ceux des noirs et des hispaniques augmentant de deux à trois points supplémentaires, ce qui traduit une amélioration et de l'efficacité et de l'équité de l'enseignement dans ces disciplines. Autre facette d'une amélioration de l'équité, cette amélioration a profité en particulier aux élèves faibles. II semble donc que les réformes aient porté quelques fruits. II s'agit là de tendances générales. Parmi les Etats qui ont implanté les politiques les plus résolues et les plus cohérentes en la matière, certains ont obtenu des résultats remarquables, tant en terme d'efficacité que d'équité, comme le Texas, la Caroline du Nord, l'Etat de New-York, tandis que, dans certains autres, notamment la Floride, ces résultats tardent à se concrétiser.

Au total, les résultats suggèrent qu'une tutelle qui combine pression sur et soutien à des ètablissements dotés d'une certaine autonomie est à même d'orienter avec une certaine efficacité leur action vers les objectifs fixés par le politique.

\section{Le bilan des politiques de choix de l'école}

Compte tenu des arguments avancés en faveur de cette politique, ce bilan porte sur la satisfaction des familles, la diversification de l'enseignement, mais surtout l'efficacité de l'enseignement et son équité.

\footnotetext{
1 Les très bons résultats du Royaume-Uni à la récente évaluation des élèves de 15 ans en compréhension de l'écrit, maths et sciences (PISA) vont dans le mème sens, sans que, pour des raisons tenant aux compétences particulières mesurées par cette étude, comme aux facteurs autres que la régulation qui ont pu jouer (le fait même qu'un curriculum national ait été adopté, par exemple), on ne puisse y voir une preuve absolue du succès de la régulation anglaise.
} 
Les familles qui ont usé de leurs possibilités de choix se montrent satisfaites, et se sont davantage impliquées dans la scolarité de leur enfant, ce qui conforte sans doute l'argument de la responsabilité.

En revanche, alors que la diversification de l'offre scolaire était un des effets attendus de la mise en marché, la concurrence a plutôt favorisé la ressemblance entre écoles, et de ce fait leur hiérarchisation et la sélection des élèves. Cela dit, dans certains pays, les écoles font parfois des choix pédagogiques réellement différents. C'est notamment le cas aux Etats-Unis, quand les curricula sont adaptés aux demandes des minorités ethniques, et pour certaines Charter schools ${ }^{1}$ qui visent des publics très particuliers.

Le choix de l'école ne peut conduire à plus d'efficacité que si les familles choisissent les établissements les plus efficaces. Or, parmi les critères déclarés par les familles, l'efficacité est effectivement citée, mais de manière moins hégémonique que cela n'était escompté par les partisans du choix. Des signes diffus d'un climat propice aux études, volontiers traditionnel, sont bien plus mobilisés par les familles, de même que des indices d'une composition sociale ou ethnique rassurante (Hunter, 1991).

Et effectivement, eu égard à la progression des élèves, qui est l'objectif essentiel, les résultats des expériences américaines restent à l'heure actuelle discutés. Certaines recherches font état de gains de productivité importants dans les écoles publiques classiques qui sont soumises à la concurrence d'une Charter school ou sous la menace de l'utilisation de vouchers par leurs élèves (Hoxby, 2001). D'autres, au contraire, pointent que les élèves qui profitent de vouchers ne réussissent pas mieux que des élèves de mêmes caractéristiques qui n'en ont pas bénéficié (Witte, 1998).

Les effets sur l'équitè sont étudiés presque exclusivement à travers la ségrégation, ethnique, sociale, plus rarement académique. Les familles de milieu populaire, en effet, peuvent se sentir concernées par les systèmes de choix, dès lors que cela leur permet d'accéder à des écoles de meilleure qualité. Certaines expériences américaines en témoignent. Mais ce sont tout de même les familles aisées et instruites (avec en tête les parents enseignants) qui utilisent le plus les possibilités de choix. Ce qui fait craindre pour l'équité, en particulier si ces familles pratiquent le "social matching".

Or, on peut s'attendre, à ce que, si ségrégation accrue il y a, elle soit assortie d'une augmentation des écarts de performance entre élèves : on sait que les élèves forts regroupés ensemble dans des écoles d'élite ont toute chance de devenir encore plus forts, alors que les élèves faibles regroupés entre eux ont toute chance d'être encore plus distancés.

De fait, la plupart des études empiriques à ce sujet, mais pas toutes, font craindre que le choix n'accroisse la ségrégation. En Belgique, la ségrégation sociale et académique est très significative (Vandenberghe, 1997). II semble bien qu'elle découle du marché, et que le nombre d'établissements

\footnotetext{
1 Les Charter schools sont des écoles dont le fonctionnement est fort peu réglementé, mais dont le contrat (Charter) peut être annulé si leurs performances sont trop faibles. Apparues en 1992, elles représentaient en $20001 \%$ des écoles américaines.
} 
accessibles au public dans un arrondissement soit un facteur qui exacerbe la ségrégation. Aux Pays-Bas, une ségrégation ethnique importante prévaut et elle s'est fortement accrue ces dernières années (Karsten, 1994). Un accroissement de la ségrégation sociale par le choix de l'école a été dénoncé également en Nouvelle Zélande (Thrupp, 1999).

Les effets du développement du choix sur l'équité du système éducatif ont fait l'objet de débats particulièrement vifs en Grande Bretagne. D'après des analyses récentes (Gorard et Fitz,1998 et 2000), entre 1988 et 1998, la ségrégation sociale entre écoles n'aurait pas augmenté; elle aurait même baissé, une légère remontée, significative, étant observée depuis les 3-4 dernières années (Noden, 2000). Mais à l'intérieur d'une zone donnée, les écoles peuvent évoluer de manière contrastée, certaines devenant les "poubelles" des autres. II semble que cela soit le cas (Gibson et Asthana,1999) : au niveau des marchés locaux, c'est à dire en situant chaque école par rapport à ses concurrents, la ségrégation est en hausse. Les écoles les mieux classées dans leur marché local sont celles qui ont vu leur niveau scolaire progresser le plus, avec par conséquent un accroissement des écarts scolaires (et sociaux) entre écoles de la même zone. Mais le fait que la ségrégation ait augmenté dans certaines zones et pas dans d'autres suggère que le choix de l'école n'a pas des effets systématiques et univoques, et la prudence reste de mise.

Cela dit, sur l'évolution de l'équité du système, l'organisation même du choix n'est pas sans importance: l'évaluation du dispositif de Minneapolis (Glazerman, 1998, cf. ci dessus), a montré que la ségrégation (notamment raciale) y est plus faible que dans un système où le choix des familles serait satisfait dans tous les cas, mais aussi plus faible que dans un système de type carte scolaire reflétant la ségrégation sociale de l'habitat.

II faut, pour finir, s'intéresser aux liens entre ségrégation et efficacité d'ensemble du système. Un certain nombre de travaux (en France, cf. DuruBellat et Mingat, 1997) montrent d'une part que ce que gagnent les élèves faibles à fréquenter une classe hétérogène est deux fois plus important que ce que perdent les meilleurs élèves à une telle situation, et d'autre part que les élèves faibles sont plus sensibles que les élèves forts à la qualité de l'environnement scolaire.

Si donc les familles les plus influentes, qui sont aussi celles dont les enfants ont en moyenne les meilleurs résultats, envoient leurs enfants dans les mêmes établissements, cela compromet les progressions des élèves qui restent dans des classes ou des établissements encore plus faibles. Les familles, en effet, prennent leur décision en fonction de bénéfices privés, mais sans voir le coût collectif de leur choix. Au total, des classes et des écoles davantage polarisées et les "effets de pairs" qui en découlent affectent à la baisse l'efficacité scolaire globale du système, alors que l'amélioration de la qualité est un des arguments les plus forts des défenseurs du choix de l'école. La question de savoir s'il est opportun de développer les possibilités de choix des familles est donc de nature politique. Si l'on privilégie l'objectif d'intégration sociale ou de "maximisation" des acquis scolaires de l'ensemble d'une classe d'âge, alors le développement du choix fera plutôt l'objet d'un jugement négatif, puisque le "marché" tend à produire de la segmentation et de la polarisation, sauf s'il est 
encadré de manière stricte. Par contre, si la satisfaction des usagers, ou encore la formation d'une élite, sont mises en avant, le jugement sera plutôt positif.

En conclusion, comme bien d'autres politiques, autonomie et choix de l'école ont des effets plus modestes que ceux qui étaient espérés ou craints. Ceux-ci s'avèrent en outre très sensibles aux dispositifs de régulation qui accompagnent leur mise en œuvre. De fait, ces politiques ne conduisent pas à un affaiblissement du rôle de l'État, mais bien à la transformation des modes de régulation publique.

Une "exception française"?
Nous comparerons d'abord la régulation en France avec le modèle que nous venons de présenter, puis nous nous interrogerons sur la consistance du modèle français: s'agit-il d'une trahison des traditions françaises au profit du modèle anglo-saxon, ou d'une adaptation au contexte français d'un modèle général. Et dans ce cas, que faut-il penser de cette adaptation?

\section{La régulation à la française}

Le modèle vers lequel convergent la plupart des systèmes éducatifs emprunte certains traits au modèle français : mise en place de programmes nationaux, importance accordée à l'instruction et aux apprentissages dans les disciplines fondamentales, examens nationaux. Cela contredit l'idée d'une adoption servile du modèle anglo-saxon, d'autant que ce modèle n'est pas le modèle traditionnel de ces pays.

Mais il est vrai que le système éducatif français, par nombre de ses évolutions, se rapproche du modèle que les pays anglo-saxons adoptent ${ }^{1}$. A partir des années 80 , on a admis en France que l'égalité ne signifiait pas l'uniformité, et on est entre dans une logique d'adaptation aux publics et d'autonomie accrue des ètablissements (Zones d'èducation prioritaires, Projet d'établissement...). Alors qu'à l'étranger on mettait davantage l'accent sur les disciplines fondamentales, on a découvert les vertus de l'interdisciplinaire, on insiste davantage sur le rôle éducatif de l'école. Parallèlement, des tentatives ont pris place pour développer l'évaluation des établissements, ou assouplir la carte scolaire. S'agissant de la régulation, pourtant, les diffèrences l'emportent sur les convergences.

Alors que dans les pays anglo-saxons, les changements sont présentés comme des réformes radicales, ils le sont en France comme des adaptations nécessaires et d'ampleur limitée. Si on exprime quelques attentes positives par rapport au développement de l'autonomie des établissements, on ne trouve dans les textes aucune défense du choix de l'école en tant que tel. Alors que dans les autres pays, les politiques de choix ont été hautement revendiquées, et hautement combattues, en France, l' " assouplissement de la

1 Pour une présentation plus détaillée de la situation en France, cf. Meuret D., Broccolichi S., Duru-Bellat M. (2001). 
carte scolaire " fut organisé, "à titre expérimental ", de manière quasi clandestine, comme s'il avait fallu concéder à l'èpoque une entorse aux principes. Il est significatif qu'on n'ait pas jugé opportun de produire un texte, loi ou décret, ce qui eut impliqué un débat national, et qu'on ait laissé aux autorités locales le soin de définir les modalités de l'assouplissement.

II résulte de cette gestion timide, négociée seulement en interne, et non pas politiquement, sur la place publique, un aspect retenu, parfois contradictoire, parfois opaque des réformes, et surtout le fait que les modes de régulation n'ont pas èté adaptés aux formes nouvelles de fonctionnement. Ceci est vrai aussi bien des politiques d'offre que des politiques de demande.

L'autonomie des établissements n'inclut pas la gestion du personnel. La participation des parents est cantonnée à la périphérie (par exemple, ils votent un projet d'établissement, mais ce projet porte sur tout sauf sur l'enseignement délivré dans les classes). On met en place des épreuves de connaissances permettant d'appréhender objectivement le niveau des élèves, mais ces épreuves servent à des fins pédagogiques et (surtout) pas à des fins d'évaluation. Plus fondamentalement, l'autonomie des établissements laisse quasi-intacte l'autonomie des enseignants, qui sont, par exemple, censés découvrir eux mêmes les mérites du travail en équipe, et l'adopter librement.

On peut trouver contradictoire que le Ministère publie des indicateurs de performances des lycées sans que les parents puissent choisir ces derniers, ou sans que rien soit entrepris envers ceux qui présentent durablement des performances faibles; que les enseignants continuent d'être évalués individuellement quant on prône le travail en équipe; que l'on multiplie les évaluations des élèves, sans évaluer les enseignants, comme si les élèves étaient seuls responsables de leurs progrès ; que, faute de pouvoir évaluer les projets d'établissements par une mesure de leurs effets, les rectorats n'aient le choix qu'entre un contrôle de conformité, qui annule l'autonomie des établissements, et un simple contrôle de légalité, qui renonce à toute régulation.

Un exemple d'opacitè est le fonctionnement des commissions chargées de réguler les demandes de dérogation. Alors qu'aux Etats-Unis par exemple, on définit de manière précise et publique les modalités du choix, en France, on confie aux commissions locales le soin d'organiser le choix dans le sens du bien commun. Si ce flou permet d'éviter les conflits sur les principes, il donne toute latitude aux fonctionnaires locaux, et davantage de pouvoir aux initiés capables de déchiffrer les critères de leurs décisions.

De fait, le système français a développé, pour accompagner l'autonomie des établissements, une forme de régulation fondée sur les processus, et non sur les résultats. L'aide et la pression s'exercent sur les ètablissements et les enseignants, non pour qu'ils améliorent leurs résultats, mais pour qu'ils mettent en oeuvre telle ou telle "forme pédagogique" ( "parcours diversifiés", par exemple), dont la rotation est aussi rapide que le bien fondé incertain. De fait, la régulation à la française repose, conformément à la notion de bureaucratie professionnelle, sur la confiance envers les professionnels, avec sans doute les conséquences qui y sont attachées selon Mintzberg, certaines malheureuses, comme la difficultè à organiser la coopération entre les acteurs, 
à traiter les problèmes inaperçus des professionnels, à gérer les cas de professionnels défaillants.

On a donc affaire à une autonomie qui n'est pas véritablement régulée ${ }^{1}$. Or l'autonomie, nous l'avons vu, n'a d'impact (notamment sur l'amélioration de la qualité de l'enseignement) que si elle est accompagnée, soit de la pression des usagers, soit, plus sûrement, de la pression de l'Etat via l'évaluation des "effets école" ou des "effets maître". Rien de cela n'existe en France. Notamment parce que la méfiance à l'encontre des usagers y est très forte. Ainsi, tout en affirmant la nécessité de développer l'information des parents, les circulaires font tout pour protéger les établissements rejetés par les usagers, décrits comme victimes des "effets souvent injustes de certains jugements rapides". La circulaire n`98-263 du 29/12/98 va jusqu'à décréter "une interdiction absolue de la concurrence entre établissements".

\section{Le bilan de la régulation française}

Concernant les effets des politiques sur le fonctionnement des établissements, plusieurs rapports de l'Inspection Générale ont montré combien les établissements peinaient à s'emparer vraiment d'une autonomie qui ne serait pas seulement formelle (se limitant à la rédaction d'un projet d'établissement conforme). Le concept même de projet d'établissement semble s'essouffler, d'autant plus que les dotations horaires sont trop contraintes pour constituer une réelle incitation à des initiatives locales. L'absence d'évaluation des actions entreprises et de pilotage des établissements sur cette base constitue un facteur supplèmentaire de désincitation.

En ce qui concerne l'"expérience" d'assouplissement de la carte scolaire, l'évolution des politiques académiques au cours des années 90 est mal connue, et l'analyse objective des effets de l'assouplissement sur la ségrégation entre établissements reste à faire. Une étude décrivant l'évolution des caractéristiques des collèges (âge et origine sociale de leur public notamment), depuis 1989, relève que les èlèves en retard, ou de milieu défavorisé, sont. un peu plus qu'avant, concentrés dans certains établissements (Trancart, 1998). Cette évolution, assez discrète, est peut-être pour une part imputable à l'assouplissement de la carte scolaire. Mais rien ne permet aujourd'hui d'établir que l'accroissement de ces disparités entre collèges est plus marqué dans les zones concernées par cet assouplissement: l'aggravation des inégalités de recrutement entre collèges est observée dans des villes où, formellement, la carte scolaire prévaut toujours (Broccholichi et van Zanten, 1997).

Enfin, il est tentant de rechercher au niveau même des performances globales du système les effets des politiques d'autonomie et de choix des établissements, sachant bien évidemment qu'on ne saurait imputer trop vite les évolutions constatées aux dites politiques. Quant aux résultats d'ensemble,

\footnotetext{
1 Un bon exemple de l'absence de régulation est que, non plus seulement le contenu de certaines «formes pédagogiques ", mais leur implantation même, sont laissés à la discrétion des enseignants (les Travaux Personnels Encadrés au lycée). Un autre est que, parfois, le fait même de suivre le programme officiel est laissé à la discrétion du professeur (en philosophie).
} 
jusqu'aux dernières évaluations internationales, les résultats du système français se comparaient favorablement avec ceux de l'Angleterre et des EtatsUnis, aussi bien en matière d'efficacité que d'équité. En termes d'efficacité, le Royaume Uni devance la France à la plus récente de ces évaluations (PISA, 2000), tandis que les Etats-Unis se sont rapprochés d'elle. Toutefois, il peut s'agir là d'un effet des différences (de populations prises en compte, de compétences mesurées) entre cette évaluation et les précédentes.

Reste la question de savoir si les politiques d'autonomie et de choix «à la française " se sont accompagnées d'une baisse ou d'une hausse de l'efficacitè du système. Les èvaluations disponibles à cet égard sont plus rares et moins systématiques qu'en Angleterre ou aux Etats-Unis. De plus, les mesures s'arrêtent en 1995. Les seules pertinentes pour notre question évoquent, pour le niveau de la troisième, une évolution semblable à celle des pays anglo-saxons : amélioration en maths, stabilité en français. Les résultats du système ne se sont donc pas effondrès, comme on aurait pu l'attendre si l'évolution avait consisté à importer un fonctionnement entièrement étranger aux traditions nationales.

Néanmoins, nombre de signes montrent que sur le plan humain, ces évolutions s'accompagnent d'un réel malaise. La dernière consultation sur le collège (cf. Dubet et Duru-Bellat, 2000) met en pleine lumière l'ambivalence que l'autonomie suscite chez les enseignants : si peu envisagent de revenir à un mode de fonctionnement plus réglè d'"en haut", nombreux sont ceux qui redoutent un éclatement du système, et des inégalités qui se creuseraient d'un établissement à l'autre. La régulation par les processus suscite le même sentiment d'atteinte à la dignité professionnelle (on nous dit comment faire!) que suscite dans les pays anglo-saxons la mesure des résultats (on nous oblige à "enseigner pour le test »!). Par ailleurs, se développe tout un discours désabusè sur les jeunes, ainsi qu'une certaine hostilité entre les enseignants et les parents.

Au total, l'origine de ce "mauvais climat" et des difficultés de fonctionnement du système français est peut-être moins technique que politique. Malgré la faiblesse des mécanismes de régulation et la rareté des évaluation ex post, le maintien d'examens et de programmes nationaux assurerait une certaine efficacité, mais la régulation incertaine du système conduirait à demander davantage à chaque individu isolément, à chaque élève comme à chaque enseignant. Par exemple, chaque enseignant isolément doit faire admettre son degré d'exigence à chaque classe séparément. Ceci vaut particulièrement dans les contextes sociaux difficiles (Van Zanten, 2001). La "crise" serait moins technique que politique, ne serait pas celle du système éducatif lui même, mais celle du "modèle éducatif " français, de la façon dont l'éducation est pensée (et rarement débattue) dans la conscience nationale. Les enseignants, qui, collectivement, rejettent les formes nouvelles de régulation au nom de ce modèle, pâtissent sans doute fortement d'une des conséquences de ce rejet: l'isolement dans lequel ils doivent exercer leur responsabilité, et, de ce fait, le caractère plus exposé de leur métier. 
Un modèle inadapté aux circonstances?

On peut lire de façon relativement optimiste l'évolution récente du système éducatif français. Il a ouvert, sans dommage pour le niveau des élèves, l'enseignement secondaire. La structure du système - un cadre national fort, une autonomie relative des acteurs - est fondamentalement saine. II suffirait donc d'introduire un peu plus d'évaluation des résultats et quelques procédures qui en tirent les conséquences.

La lecture pessimiste s'appuie sur les effets pervers de réformes visant à mettre "l'élève au centre du système", engageant à entrer dans une logique de "service du public", au sein d'un modèle qui y est fondamentalement opposé.

Le système éducatif français traditionnel vise en principe l'émancipation par la raison. Un tel projet est à double face: il s'agit d'une part de promouvoir l'individu libre, de l'arracher aux traditions, et en ce sens il s'agit d'un projet de libération. Mais, dans un autre sens, il s'agit d'un procès de domestication d'un enfant dont on suppose que l'environnement non scolaire est inapte à le socialiser. Cette vision de l'"Etat éducateur" procède elle-même d'une conception de l'Etat plus proche de Rousseau que de Locke ou de Smith : II promeut, en lieu et place du heurt " naturel » des intérêts, une société civile fondée sur la citoyenneté. Dans ce cadre, l'éducation délivre des brevets de citoyenneté, ce qui rend particulièrement infamant l'échec scolaire. Par exemple, dans un discours sur le collège d'avril 2001, le Ministre définit l'éducation « le garant de l'unité de la République ». Elle garantit que " tous les enfants de France pourront, le moment venu, jouer leur rôle de citoyen ", et ses objectifs s'organisent, non pas autour du développement de chaque enfant, non pas autour de sa capacité à mener une vie riche et responsable, mais autour de la capacité de tous les enfants "à se parler et à se comprendre, à communiquer dans la sérénité ». A l'inverse, un ouvrage américain de philosophie politique de l'éducation (Gutman, 1999) pose le problème ainsi : "How should citizens be educated, and by whom ? "ce qui signifie que ce n'est pas de l'éducation que les enfants tiennent leur qualité de citoyen, mais de leur appartenance à la communauté.

On peut avancer que, dans le cadre de ce modèle, l'État peut se dessaisir de certaines prérogatives au profit des professionnels, ce qu'il a fait, mais non au profit des usagers.

Or, deux tendances lourdes font que les usagers ne pourront que réclamer un rôle plus important. D'une part la pression de l'école sur les individus s'est sensiblement accrue, et d'autre part l'éducation est devenue davantage un bien privé. Aujourd'hui, l'emprise de l'école semble écrasante : les familles de tous milieux (Lahire, 1995), les institutions (justice, police, armée, média, ..) ou encore le patronat comptent sur elle et la soutiennent. S'ils la critiquent, c'est seulement qu'elle serait infidèle à sa mission ou inefficace dans son accomplissement. Ainsi, l'indignité vis à vis de l'école est de plus en plus une indignité globale. La violence faite aux élèves faibles liée au modèle français, supportable tant que l'école était une des multiples dimensions de la vie en société, devient insupportable quand elle les résume toutes. 
Par ailleurs, ce savoir que l'école transmettait pour introduire à un ordre plus haut que celui du monde, on s'attend aujourd'hui à ce qu'il soit utile à la vie dans le monde. L'éducation est de moins en moins un bien public, et, de plus en plus, un "bien privé à concernement collectif " (Bénard, 1985), soit un bien privé, mais affecté de multiples externalités. En tant que bien privé, l'éducation fait l'objet d'une demande. En tant que bien à concernement collectif, elle ne peut être laissée au soin des individus et du marché, mais il reste qu'elle ne peut plus être conçue d'abord comme un bien en quelque sorte imposé par l'État au nom du bien public.

\section{Conclusion}

L'évolution récente du système français n'est pas le résultat d'une domination du modèle anglo-saxon, et d'abord parce que ce dernier s'est réformé lui même, y compris en empruntant au système français ; ensuite, parce que, si l'on avait importé tel quel un modèle fondé sur des traditions étrangères, des dysfonctionnements majeurs n'auraient pas manqué d'apparaître.

II nous semble qu'il y a bien dans l'idée d'établissements autonomes jugés d'après leurs résultats une forme de rationalisation, que la France a essayé de combiner avec son propre modèle d'éducation, sans vraiment y réussir. Pourquoi ?

Si cette analyse est correcte, la réforme qui s'est opérée dans les pays anglosaxons était certes contraire à la culture des enseignants, à qui on a demandé de s'intéresser moins à l'éducation et plus à l'instruction, mais elle n'était pas contraire au modèle éducatif de ces pays, lequel stipule qu'il faut préparer les enfants pour continuer et renouveler le monde, que les enseignants doivent assurer la responsabilité de ce monde $\mathrm{ci}$, et non être les témoins d'une République idéale ${ }^{1}$. II suffisait d'argumenter, ce qui a été fait, que la réforme permettait mieux aux élèves d'affronter la vie qui les attendait.

A l'inverse, dans le modèle français, l'éducation institue la République. Or, dans le cadre d'une éducation instituante, la charge de la preuve est toujours du côté de l'élève, jamais du côté du système, de ses agents, de ses institutions. L'intervention des usagers, la mesure des résultats sont profondément contraires à la logique du système. II se trouve cependant que ce modèle n'est plus adapté à un monde où la tâche de l'Etat est de réguler d'une façon efficiente, favorable à la justice sociale et à la vivacité démocratique, la production d'un bien (l'éducation) auquel tous aspirent et qu'ils sont prêts à aller chercher ailleurs que dans les institutions d'Etat.

Abandonner le modèle d'une éducation instituante ne signifie pas que l'on tombe dans une logique de marché. D'une part, même le choix de l'école peut être régulé par l'Etat de façon à produire les effets désirés. D'autre part, la participation des usagers peut passer par l'expression de leur avis et non par

\footnotetext{
1 On soutient ici que le système éducatif américain est plus proche du modèle qu'H. Arendt (1989) trace pour lui que de la caricature qu'elle en présente elle-même, alors que le modèle français, qui refonde la République à chaque génération, est, malgré certaines apparences, fort éloigné du modèle arendtien qui fait des éducateurs les « représentants du monde, dont ils doivent assumer la responsabilité, même (...) s'ils le souhaitent différent de ce qu'il est ».
} 
le choix, II va de soi cependant que renoncer au choix suppose une politique résolue d'actions en direction des établissements et enseignants en difficulté, qui se trouve aussi empêchée par le modèle.

L'enjeu actuel est de retrouver la possibilité d'un gouvernement de l'école, et cela passe par un compagnonnage moins conflictuel entre la société et son école, qui se traduirait par une régulation démocratique de l'éducation, où l'Etat aurait charge de faire émerger ce que la société attend de l'école, de sorte que ses objectifs ne soient définis ni par les seuls besoins de l'économie, ni par la seule "offre" proposée par les enseignants, ni par l'agrégation des demandes individuelles. Sans doute ce débat et de manière plus durable ce compagnonnage lui-même doivent-ils être organisés : à l'heure actuelle, la faiblesse des associations laisse face à face l'État et les individus; la participation des catégories populaires y est loin d'être acquise, et pourtant essentielle.

Tout ceci exige, pour advenir et perdurer, l'instauration si ce n'est d'un nouveau modèle aussi fort que l'ancien, du moins d'un "nouveau référentiel de l'action publique" (pour reprendre la formule de Jobert, 1992), proposant une nouvelle manière de modéliser la vocation de l'institution scolaire, de la rendre intelligible et légitime. 


\section{BIBLIOGRAPHIE}

ARENDT H. (1989), La crise de l'éducation, in La crise de la culture, Paris : Folio, (trad. fcse).

BENARD J. (1985), Economie Publique, Paris, Economica.

BROCCHOLICHI S., VAN ZANTEN A. (1997), Espaces de concurrence et circuits de scolarisation, Annales de la recherche urbaine, $n^{\circ} 75,5-17$.

CARL J. (1994), Parental Choice as National Policy in England and the United States, Comparative Education Review, 38, 3, 294-323.

Center on Education Policy (2000), The Good News about American Education, Washington, $34 \mathrm{p}$.

CHUBB J.E. et MOE T.M. (1990), Politics, Markets and America's Schools. Washington: The Brooking Institution.

Condition of education (1998), US Department of Education, Office on Education Research and Improvement, National Center of Educational Statistics, $369 \mathrm{p}$.

DUBET F. et DURU-BELLAT M. (2000), L'hypocrisie scolaire, Paris : Seuil. DURU-BELLAT M. et MINGAT A. (1997), La constitution de classes de niveau par les collèges et ses incidences sur les progressions et les carrières des élèves. Revue française de sociologie, XXXVIII, 4, 759-790.

DURU-BELLAT M. et MEURET, D.,2001, Nouvelles formes de régulation dans les systèmes éducatifs étrangers: autonomie et choix des établissements scolaires, Revue Française de Pédagogie, №135, 173-221.

Education week, 2001, Seeking stability for standard-based education, Quality Counts 2001, disponible sur www.edweek.org/sreports/gc01.

FINKELSTEIN N.D. et GRUBB W.N., 2000, "Making sense of Education and Training markets : Lessons from England", American Educational Research Journal, 37(3), 601-632.

GIBSON A., et ASTHANA S. (1999), Schools, Markets and Equity ; access to secondary education in England and Wales, Communication au congrès de l'American Educational Research Association, Montréal, 19-23/04.

GLAZERMAN S. (1998), School Quality and Social Stratification : The Determinants and Consequences of Parental Choice. Communication au congrès de l'American Educational Research Association, San Diego.

GORARD S., \& FITZ J. (1998), The More Things Change... The Missing Impact of Marketisation, British Journal of Sociology of Education, 19, 3, 365-375.

HERRINGTON C., KASTEN K. (2000), Educational Policy Alternatives, Technical Report, 229 p., Florida 2001.

HOXBY C. (2001), School choice and School productivity (or, could school choice be a tide that lifts all boats ?), NBER, Paper for the conference on the Economics of School Choice, $73 \mathrm{p}$.

HUNTER J. (1991), Which school ? A study of parents'choice of secondary school, Educational Research, 33, 1, 31-41.

JOBERT B. (1992), Représentations scciales, controverses et débats dans la conduite des politiques publiques, Revue Française de Sciences Politiques, vol. 42, 219-233.

KARSTEN S. (1994), Policy on ethnic segregation in a system of choice : the case of The Netherlands, Journal of Education Policy, 9, 3, 211-225. 
LAHIRE B. (1995), Tableaux de familles Heurs et malheurs scolaires dans les milieux populaires, Paris, Gallimard-le Seuil.

LEE V.E. \& SMITH J.B. (1995), Effects of high school restructuring and size on early gains in achievement and engagement, Sociology of Education, 68, 241-270.

LEIGHTWOOD K \& MENZIES T.(1998), A review of research on School based management, School Effectiveness and School Improvement, 9, 3, 233285

MEURET, D., BROCCOLICHI, S., DURU-BELLAT, M. (2001), Autonomie et choix des établissemients scolaires: finalités, modalités, effets, Cahier de I'IREDU, $\mathrm{n}^{\circ}$ 62, Dijon, $262 \mathrm{p}$.

MEURET D. et DURU-BELLAT M. (2001), A comparison of the English and French regulations of the Educational systems, Communication à l'European Congress of Educational Research, Lille.

MURPHY J., \& BECK L.G. (1995), School Based Management as School Reform, Corwin Press, A Sage publication, $216 p$.

NODEN P. (2000), Rediscovering the impact of marketisation : dimensions of social segregation in England's secondary schools, 19941999, British Journal of Sociology of Education, 21, $n^{\circ} 3,371-390$.

OCDE-CERI (1998), Regards sur l'éducation, Paris.

SCHRIEWER J., 1997, L'éducation comparée : Mise en perspective historique d'un champ de recherche, Revue Française de pédagogie, $\mathrm{n}^{\circ} 121,9-28$.

TEELKEN C. (1999), Market Mechanisms in Education: School Choice in the Netherlands, England and Scotland in a Comparative Perspective. Comparative Education, 35, 3, 283-302.

THRUPP M. (1999), School making a difference : Let's be realistic, Buckingham : Open University press.

TRANCART D. (1998), L'évolution des disparités entre collèges publics, Revue Française de Pédagogie, $n^{\circ} 124,43-53$.

VANDENBERGHE V. (1997), Le dispositif institutionnel scolaire belge : un mécanisme hybride alliant l'état et le marché, marqué par la ségrégation entre écoles, Savoir, 9, 3-4, 363-375.

VAN ZANTEN A. (2001), L'école de la périphérie, Paris, Seuil, 424 p.

VITERITTI J.P. (1999), Choosing equality : School choice, the Constitution and Civil society, Brookings Institution, $284 \mathrm{p}$.

WITTE J.F. (1998), The Milwaukee Voucher Experiment, Educational Evaluation and Policy Analysis, 20, 4, 229-251.

WÖSSMANN L. (2000), Schooling Ressources, Educational Institutions and student performance : the international evidence, Kiel Working paper $\mathrm{n}^{\circ} 983$, Kiel Institute for World Economics, $86 \mathrm{p}$. 